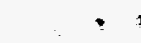

LA-UR/LA=OP-98-

Approved for public release;

distribution is unlimited.
4. 4 年

TITLE:

MODELING OF WATER RADIOLYSIS AT SPALLATION NEUTRON SOURCES

AUTHOR(S):

L.L. Daemen, LANSCE 12

G.S. Kanner, MST-6

R.S. Lillard, MST-6

D.P. Butt, MST-6

T.O. Brun, LANSCE 12

W.F. Sommer, APT-TPO

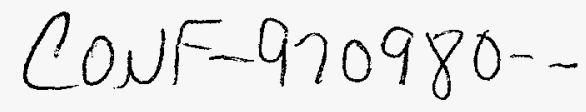

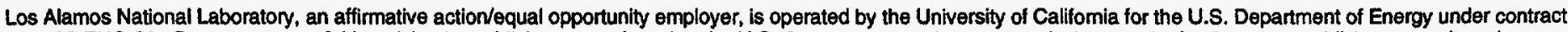

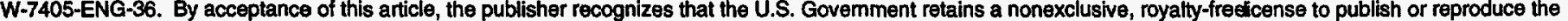

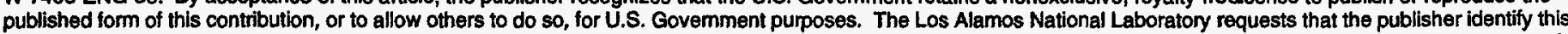

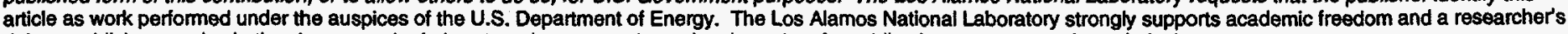
right to publish; as an institution, however, the Laboratory does not endorse the viewpoint of a publication or guarantee its technical correctness. 


\section{DISCLAIMER}

This report was prepared as an account of work sponsored by an agency of the United States Government. Neither the United States Government nor any agency thereof, nor any of their employees, makes any wartanty, express or implied, or assumes any legal liability or responsibility for the accuracy, compieteness, or usefulness of any information, apparatus, product, or process disclosed, or represents that its use would not infringe privately owned rights. Reference herein to any specific commercial product, process, or service by trade name, trademark, manufacturer, or otherwise does not necessarily constitute or imply its endorsement, recommendation, or favoring by the United States Government or any agency thereof. The views and opinions of authors expressed herein do not necessarily state or reflect those of the United States Government or any agency thereof. 


\section{DISCLAIMER}

Portions of this document may be illegible in electronic image products. Images are produced from the best available original document. 


\title{
MODELING OF WATER RADIOLYSIS AT SPALLATION NEUTRON SOURCES
}

\author{
L.L. Daemen (1), G.S. Kanner ${ }^{(2)}$, R.S. Lillard ${ }^{(2)}$, D.P. Butt ${ }^{(2)}$, T.O. Brun ${ }^{(1)}$, and W.F. Sommer ${ }^{(3)}$ \\ (1) Manuel Lujan, Jr. Neutron Scattering Center \\ (2) Materials Corrosion and Environmental Effects Laboratory \\ (3) Ceramic Science and Technology \\ Los Alamos National Laboratory \\ Los Alamos, New Mexico 87545
}

\section{Abstract}

In spallation neutron sources neutrons are produced when a beam of high-energy particles (e.g., $1 \mathrm{GeV}$ protons) collides with a (watercooled) heavy metal target such as tungsten. The resulting spallation reactions produce a complex radiation environment (which differs from typical conditions at fission and fusion reactors) leading to the radiolysis of water molecules. Most water radiolysis products are short-lived but extremely reactive. When formed in the vicinity of the target surface they can react with metal atoms, thereby contributing to target corrosion. We will describe the results of calculations and experiments performed at Los Alamos to determine the impact on target corrosion of water radiolysis in the spallation radiation environment. Our computational methodology relies on the use of the Los Alamos radiation transport code, LAHET, to determine the radiation environment, and the AEA code, FACSIMIILE, to model reaction-diffusion processes

\section{Intraduction}

Nuclear reactor facilities have long recognized that exposure of materials to cooling water that has been subjected to intense radiation fields results in enhanced corrosion rates [1]. The presence of the radiation field, primarily neutrons and high-energy photons at fission reactors leads to water radiolysis which, in tum leads to enhanced corrosion of metals in contact with the irradiated water. The radicals and ions formed during water radiolysis, though usually short-lived, can be extremely reactive. Because of their short lifetime, their effect is limited to the immediate vicinity (within a few diffusion lengths) of the point in space where they are generated. The main long-lived products of water radiolysis are $\mathrm{O}_{2}, \mathrm{H}_{2}$, and $\mathrm{H}_{2} \mathrm{O}_{2}$. They are all soluble in water, and, because of their stability, their concentration builds up over time. They are readily transported to all locations in the cooling system and can affect material corrosion anywhere in it. Solutions have been developed to mitigate the problem at fast and thermal reactors. A typical solution consists in adding reducing agents, such as hydrogen, to the cooling water. Unfortunately, this affects the physical and chemical properties of the cooling water, thereby causing other problem, and it is fair to say that the control of water chemistry and corrosion at nuclear facilities remains a delicate balancing act.

A similar problem presents itself at spallation neutron sources (accelerator-driven neutron sources) where neutrons are produced when a beam of high-energy particles (e.g., protons or deuterons) hits a heavy-metal target (e.g., tungsten or lead). Typical beam energies are in the $1 \mathrm{GeV}$ range at existing sources; the beam power is typically tens of $\mathrm{kW}$. The energy deposited in the target material is substantial and requires active cooling (with light- or heavy-water) of the target assembly. From an operational standpoint, radiation-enhanced corrosion has not been a major problem at existing spallation neutron sources, although there is some evidence that it occurs. This probably does not come as a surprise given the relatively low powers at which these sources operate. (The most powerful spallation neutron source in existence today is ISIS at the Rutherford-Appleton Laboratory near Oxford, UK, with a beam power of $180 \mathrm{~kW}$.) Several projects in the US, Europe, and Japan, however, are considering the construction of more powerful spallation neutron sources for various applications such as neutron scattering and radiography, transmutation of nuclear waste, and the production of tritium. The Accelerator Production of Tritium (APT) project is currently considering designs with beam powers of up to $200 \mathrm{MW}$, or roughly three orders of magnitude beyond the present experience.
It is rather clear that at the power levels of a project such as APT, water radiolysis and radiation-enhanced corrosion (among other materials problems) are going to emerge as major materials-related issues. Unfortunately, because of the drastically different nature of the radiation environment at a nuclear reactor and at a spallation neutron source, the broad experience base acquired for radiation-enhanced and water chemistry at reactors does not apply directly, if at all, to the spallation neutron source case. Given the cost and complexity of replacing failed spallation targets, it is important to gain some understanding of the processes responsible for water radiolysis and radiation-enhanced corrosion in the spallation radiation environment. This is the main purpose of a program initiated at Los Alamos National Laboratory in 1996. The program has two main components, namely the modeling of the water radiolysis and corrosion processes in the spallation radiation environment, and the experimental study of water radiolysis and corrosion phenomena at the LANSCE $(0.8 \mathrm{MW})$ linear accelerator. This document focuses on the prediction of water radiolysis products generation rates from computer models.

More precisely, we developed a computational methodology which starts from the radiation environment generated from a computer model of the target station and uses this information, with experimentally measured cross-sections for water excitation, ionization, and dissociation to predict the rate of generation of short- and long-lived radiolysis products. The next section gives a brief overview of the water radiation chemistry that is relevant to the spallation radiation environment. It is followed by a section describing computer models, computer codes and computational methodology for the study of water radiolysis. We illustrate the use of this methodology with a model developed for the APT project in the following section. The last section states our conclusions.

\section{A Primer on Water Radiation Chemistry}

Many excellent books and review articles are available on radiation chemistry [2]. Much progress has been made in recent years in our understanding of the microscopic aspects of water radiolysis. Our purpose here is not so much to add to this body of knowledge as it is to use this information to predict the nature and rate of formation of those radiolysis products of concern to the target engineers. Therefore, rather than modeling radiolysis phenomena in considerable detail, e.g., viā Monte-Carlo techniques, we will adopt a more pragmatic approach and rely on experimental cross-sections and simplified models to produce results in a timely fashion. This is in many ways the approach adopted in many familiar radiation transport codes.

In this section, we summarize information pertinent to the computer modeling of water radiolysis products. We start by describing the effect of the passage of various charged and neutron particles in water and discuss the formation of tracks, particularly electron tracks. This is followed by a brief description of the various possible outcomes of the collision between a water molecule and an electron.

\section{Spallation}

For the sake of definiteness, we will consider the situation of a proton accelerator bombarding some water-cooled heavy-metal target in what follows. A typical proton energy for the APT project is $1.7 \mathrm{GeV}$. These protons will suffer nuclear reactions with nuclei in the target. These socalled spallation reactions proceed in two stages: cascade and evaporation [3]. During the cascade phase, nucleons and mesons are 
ejected from the nucleus; the ejected particles can have enough energy to induce secondary spallation reactions. During the subsequent evaporation phase, the remaining excited nucleus relaxes to its ground state by emitting ("evaporating") mostly neutrons and photons. The radiation environment of a spallation neutron source has therefore many components: protons, pions, muons, neutrons, photons, and recoiling nuclei to cite but the most important. These particles can all lead to water radiolysis as explained next.

\section{Interaction of charged and neutral particles with water.[4]}

Protons and other charged particles: Charged particles lose energy by Coulomb interaction with the electrons in the atoms of the medium (e.g. water). Fast, heavy particles usually lead to the formation of secondary electrons with energies ranging from a few $\mathrm{eV}$ to a few $\mathrm{MeV}$ by atom ionization. The secondary electrons can themselves interact with the atoms in the medium; they have sufficiently low energies to lead to the excitation (rotation, vibration) of water molecules, to the formation of excited electronic states, or to the ionization of water molecules. In some cases, the excited water molecule can subsequently dissociate into a variety of neutral (radicals) or ionized products. Charged particles such as protons, muons, electrons, and charged recoiling atoms interact with water in this manner.

Neutrons: Neutrons cannot directly interact with electrons in molecules and atoms. If they have enough energy, they can collide with individual nuclei and produce a charged recoiling atom. This recoiling atom interacts with water as described above.

Photons-: Photons can interact directly with electrons in atoms and molecules by means of the electromagnetic interaction. Photoelectric excitation or ionization, Compton scattering, and pair production (from high-energy photons) are ways in which photons create low-energy secondary electrons which can then interact with water molecules, as described above.

To summarize, the various components of the spallation radiation field end up creating low-energy secondary electrons which, in fine, are responsible for water radiolysis. It is thus clear that a first step in our calculation of water radiolysis in the spallation environment will involve, at the very least, the determination of $(\alpha)$ the radiation environment, $(\beta)$ the secondary, low-energy electrons formed product by the various components of the radiation environment, and $(\gamma)$ water radiolysis products resulting from the interetion of the secondainy electrons with water molecules. We will diseess (a) will be bilefly in the section on "Computational Methodology" where we will describe some. well-known radiation transport codes. Several empirical and semi exist to describe the formation of secondary electrons by charged and neutral particles, (B), whereas a large amount of information, theoretical and experimental, is available regarding the electron-molecule collisions in the case of water, $(\gamma)$.

\section{Eormation of secondary electron}

Charged particles are most likely to lead to direct ionization of water molecules, and we will therefore consider only this mode of creation of secondary electrons in what follows. The differential cross-section describing the creation of secondary electrons by an incident (primary) electron with energy $E$ has been measured experimentally by Opal et al [5]. The data is well fitted by a set of two semi-empirical formulas proposed by Green and Sawada [6]. The cross-section for the production of an electron of energy $E^{\prime}$ by an incident electron of energy E is:

$$
\begin{aligned}
S\left(E, E^{\prime}\right)=\sigma_{0} & \frac{K}{E} \ln \left(\frac{E}{J}\right)\left[\frac{\Gamma(E)^{2}}{\left(E^{\prime}-E_{0}(E)\right)^{2}+\Gamma(E)^{2}}\right] \\
E_{0}(E) & =E_{s}-\frac{E_{2}}{E+2 I}, \\
\Gamma(E) & =\Gamma_{s} \frac{E}{E+I} .
\end{aligned}
$$

where $\sigma_{0}=10^{-16} \mathrm{~cm}^{2}$ is a cross-section scaling factor. The other parameters were determined by a fit to experimental data. For water, $\mathrm{K}=2.78 \mathrm{eV}, \mathrm{J}=0.0309 \mathrm{eV}, \mathrm{E}=1.28 \mathrm{eV}, \mathrm{E}=12.6 \mathrm{eV}, \Gamma=12.8$, and $\mathrm{I}=12.6$ eV [6]. (This last quantity is the ionization threshold for water.) Fig.1 shows $S\left(E, E^{\prime}\right)$ versus $E^{\prime}$ for several values of $E$. Berger [7] derived a simple result for the polar angle of the primary and of the secondary electron:

$$
\begin{gathered}
\sin ^{2} \vartheta_{p}=\frac{\left(E^{\prime} / E\right)}{\left(1-E^{\prime} / E\right) E /\left(2 m c^{2}\right)+1}, \\
\sin ^{2} \vartheta_{s}=\frac{\left(1-E^{\prime} / E\right)}{1+E^{\prime} /\left(2 m c^{2}\right)} .
\end{gathered}
$$

These simple formulas have been verified extensively. However, little data is available for the case when the incident (primary) particle is not an electron. (The case of an energetic proton is the most important for us.) Garcia [8] has shown that one can continue to use the above formulae with the replacement of the proton by an "equivalent" electron. The above formula remains valid if we replace an incident proton with velocity $v$ and mass $m_{p}$ (and kinetic energy $E_{p}=m_{p} v_{p}{ }^{2} / 2$ ) by an "equivalent" electron of mass $m$ with the same velocity and therefore, with kinetic energy $\mathrm{E}=(\mathrm{m} / \mathrm{m}) \mathrm{E}_{\mathrm{p}}$. This scaling relation is well confirmed by experiment results that show that the experimental ionization cross-section (and stopping power) depends on the charge and velocity of the ionizing particle, but not on its mass.

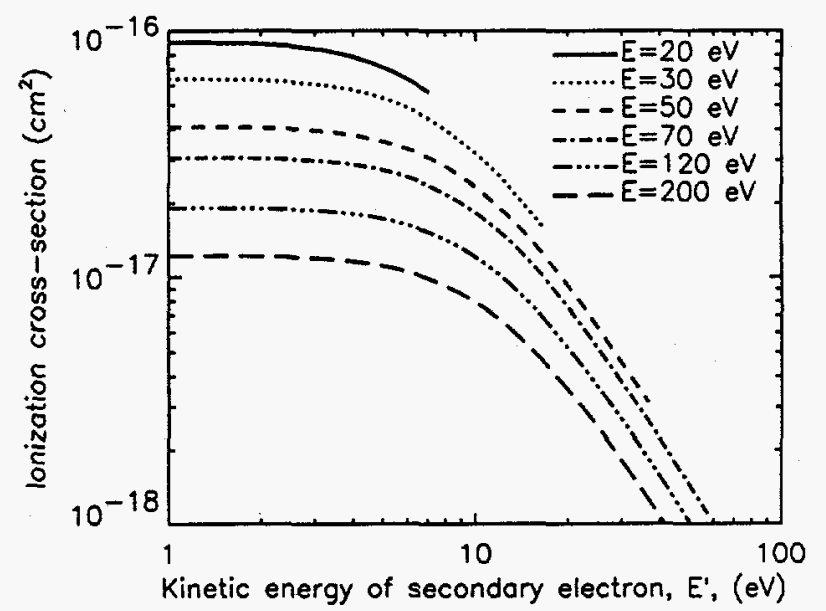

Figure 1: Cross-section for the production of secondary electrons for several values of the energy of the primary electron.

The above formulae were used in our calculations to generate secondary electrons from primary protons and electrons. (Secondary electrons can themselves generate more secondary electrons.) Even though these formulae were applied for protons and electrons, there is an important qualitative difference between these two situations. Elementary kinematics considerations show that an electron can lose up to half its kinetic energy in a collision with another electron (and, hence, can communicate only half of its kinetic energy to the ejected electron, $\left.\mathrm{mv}^{2} / 4\right)$. A heavy particle such as a proton, with the same initial velocity, on the other hand, can communicate to an electron an energy up to $2 \mathrm{mv}$ while losing only a tiny fraction, $\mathrm{m} / \mathrm{m}_{\mathrm{p}}$ of its kinetic energy. This will result, as we shall see later, in quite different track structures for electrons and protons.

Other models exist to generate secondary electrons in water. In particular, let us mention the model developed by the Notre Dame group and based on experimentally determined dipole oscillator strength distributions [9].

\section{Excitation ionization, and dissociation of water molecules}

After generating secondary electrons, the next step consists in determining the result of their collision with water molecules. The physics of these collisions is extremely complex and has been studied extensively over the past 40 years or so. It is possible to group these physical phenomena into various classes, [10].

Vibrationalexcitations: These are low-energy excitations. They play no direct role in water radiolysis. Their importance lie in that they provide a 
mechanism whereby energy from very low-energy electrons is absorbed by the medium. Typically, electrons which have lost so much energy that their kinetic energy is below the ionization threshold will lose their remaining energy by exciting vibrational levels. We modeled the crosssection for the seven most important vibrational excitations using an analytical representation proposed by Dayashankar et al.[11]

$$
\begin{aligned}
& \sigma_{\text {vib }}(E)=\sigma_{0} \frac{A}{W^{2}}\left(\frac{W}{E}\right)^{p}\left(\ln \frac{E}{W}\right)^{q} \\
& \quad+\sigma_{0} \frac{A^{\prime}}{W^{\prime}}\left(\frac{W}{E}\right)^{p^{\prime}}\left(\ln \frac{E}{W}\right)^{q^{\prime}},
\end{aligned}
$$

where $\mathrm{E}$ is the energy of the primary electron, $\sigma_{0}=10^{-16} \mathrm{~cm}^{2}$, and the parameters, $A, W, p, q, A^{\prime}, W^{\prime}, p^{\prime}$, and $q^{\prime}$ were obtained by fittings the above expression to the results of Seng and Linder [12] and Trajmar et al. [13]. These parameters are given in Table I. The cross-sections for vibrational excitations are shown in Fig.2. The remaining vibrational states were lumped together in an eighth curve also shown in Fig.2.

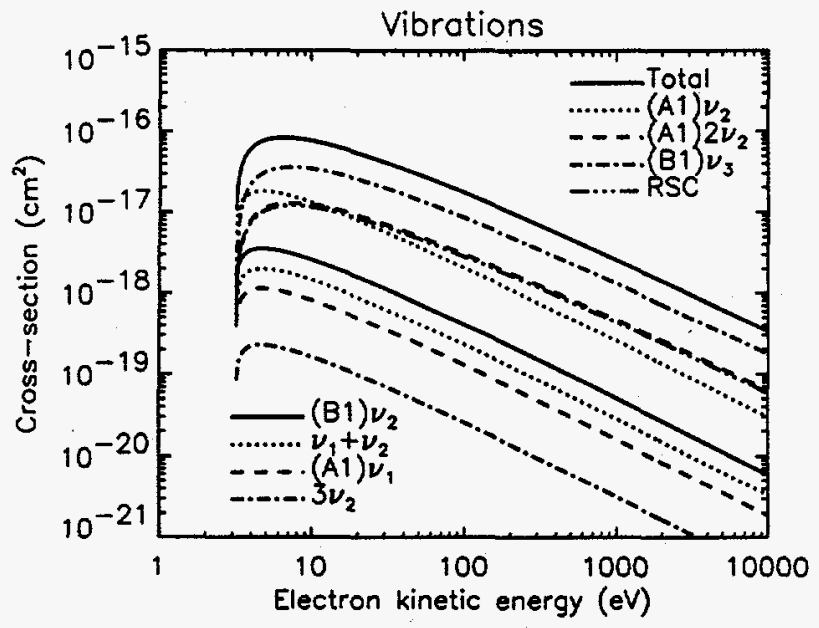

Figure 2: Cross-sections for vibrational excitation of water. RSC stands for "Remaining States Combined"

\begin{tabular}{|l|c|c|c|c|c|}
\hline & $W(\mathrm{eV})$ & $\mathrm{A}\left(\mathrm{eV} \mathrm{V}^{2}\right)$ & $\mathrm{p}$ & $\mathrm{A}^{\prime}(\mathrm{eV})$ & $\mathrm{g}^{2}$ \\
\hline$(\mathrm{A} 1) v_{2}$ & 0.198 & 2.3 & 4 & 3.979 & 0.417 \\
$(\mathrm{~A} 1) 2 v_{2}$ & 0.391 & 0.144 & 4 & 0.248 & 0.417 \\
$(\mathrm{~A} 1) v_{1}$ & 0.453 & 19.45 & 8 & 3.520 & 0.833 \\
$(\mathrm{~B} 1) v_{3}$ & 0.466 & 54.23 & 8 & 9.831 & 0.833 \\
$3 v_{2}$ & 0.579 & 0.028 & 4 & 0.049 & 0.417 \\
$v_{1}+v_{2}$ & 0.648 & 2.487 & 6 & 0.442 & 0.417 \\
$(\mathrm{~B} 1) v_{2}+v_{3}$ & 0.661 & 4.440 & 6 & 0.788 & 0.417 \\
$\mathrm{RSC}$ & 1.0 & 18.15 & 8 & 3.289 & 0.833 \\
\hline
\end{tabular}

Table I: Parameters for water vibrational cross-sections, Eq.(6). For all states: $q=2, W^{\prime}=3.2 \mathrm{eV}$, and $p^{\prime}=1$.

lonization: Ionization of the water molecule can result in its dissociation. The nature of the dissociation products depends on the molecular energy levels configuration the water molecule ends up in. Five levels can be identified; they are listed in Table II together with the corresponding energy and dissociation products [14]. In Fig. 3 we show the crosssections for the formation of the radiolysis products resulting from ionization. The curves are represented analytically by a simple expression similar to Eq.(1), $[6,10,15]$ :

$$
S\left(E, E^{\prime}\right)=\sigma_{0} \frac{K_{a}}{E+K_{b}} \ln \left(\frac{E}{J_{a}}-J_{b}\right)\left[\frac{\Gamma_{0}^{2}}{\left(E^{\prime}-E_{0}\right)^{2}+\Gamma_{0}^{2}}\right]
$$

where the extra parameters are necessary to fit the distribution of $E$ and $E^{\prime}$ for the various ionization continua. As before, $\sigma_{0}=10^{-16} \mathrm{~cm}^{2}, E$ is the energy of the incident electron, and $E^{\prime}$ is the energy of the secondary electron. The parameters are given in Table III, [10]. Eq.(7) can be integrated over E' to give an ionization cross-section for each ionization continuum:

$$
\begin{aligned}
\sigma_{\text {ion }}(E)=\sigma_{0} & \frac{K_{a}}{E+K_{b}} \ln \left(\frac{E}{J_{a}}-J_{b}\right) \\
& \Gamma_{0}\left[\arctan \frac{(1 / 2)(E-I)-E_{0}}{\Gamma_{0}}+\arctan \frac{E_{0}}{\Gamma_{0}}\right],
\end{aligned}
$$

where I is an ionization energy. (In Eq.(7), the kinetic energy of the incident electron after ionization, E-E'-I, must be positive.)

\begin{tabular}{|c|c|c|}
\hline Leve & Energy (eV) & Radiolysis Products \\
\hline $1 \mathrm{a}_{1}$ & 539.7 & $\mathrm{O}^{+}, \mathrm{H}^{+}$ \\
$2 \mathrm{a}_{1}$ & 32.4 & $\mathrm{H}^{+}, \mathrm{O}^{+}$ \\
$1 \mathrm{~b}_{2}$ & 18.51 & $\mathrm{OH}^{+}, \mathrm{H}^{+}, \mathrm{O}^{+}$ \\
$3 \mathrm{a}_{1}$ & 14.75 & $\mathrm{H}_{2} \mathrm{O}^{+}$ \\
$1 \mathrm{~b}_{1}$ & 12.62 & $\mathrm{H}_{2} \mathrm{O}^{+}$ \\
\hline
\end{tabular}

Table II: Ionization levels for water and corresponding radiolysis products.

\begin{tabular}{|c|c|c|c|c|c|}
\hline & $\mathrm{K}_{2}(\mathrm{eV})$ & $\mathrm{K}_{\mathrm{h}}(\mathrm{eV})$ & $\mathrm{J}_{2}(\mathrm{eV})$ & $\mathrm{J}_{\mathrm{h}}(\mathrm{eV})$ & $T(\mathrm{eV})$ \\
\hline $\mathrm{H}_{2} \mathrm{O}^{+}$ & 4.05 & 110.0 & 1.0 & -30.0 & 12.62 \\
$\mathrm{OH}^{+}$ & 1.134 & 94.0 & 1.0 & 0 & 20.0 \\
$\mathrm{H}^{+}$ & 1.55 & 147.0 & 1.0 & 18.0 & 20.0 \\
$\mathrm{O}^{+}$ & 0.12 & 90.5 & 1.0 & 19.0 & 20.0 \\
$\mathrm{O}^{++}$ & 0.0073 & 78.0 & 2.86 & 20.0 & 60.0 \\
$\mathrm{H}_{2}^{+}$ & 0.0091 & 29.4 & 1.0 & 0.0 & 23.0 \\
\hline
\end{tabular}

Table III: Parameters for water ionization cross-section, Eqs.(7) and (8). For all radiolysis products, $\mathrm{E}_{0}=-2.37 \mathrm{eV}$ and $\Gamma_{0}=11.4 \mathrm{eV}$.

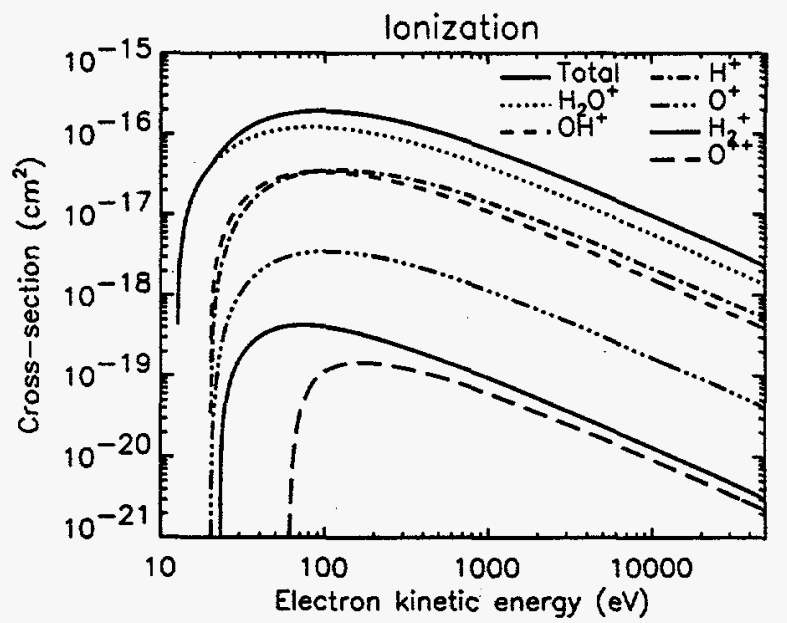

Figure 3: Cross-section for the production of radiolysis products from ionization by electrons in water.

Dissociative excitations: We consider two classes of dissociative excitations. First, there are two states, labeled $A^{1} B$, and $B^{1} A$ corresponding to dissociative continua. They are non-Rydberg states and the associated cross-section can be represented analytically as follows [14]:

$$
\sigma_{\text {diss.con. }}(E)=q_{0} \frac{A}{W^{2}}\left(\frac{W}{E}\right)^{\Omega}\left[1-\left(\frac{W}{E}\right)^{\beta}\right]^{v} \text {, }
$$

where $\mathrm{E}$ is the primary electron energy, and $\mathrm{q}_{0}=6.514 \times 10^{-18} \mathrm{~m}^{2} \cdot \mathrm{eV}^{2}$. The parameters are given in Table IV; they were taken from Ref.[15] which list the values of the parameters for liquid water rather than from Ref.[10] where they are given for water vapor. The dissociative continuum around $7.5 \mathrm{eV}$ leads to the formation of $\mathrm{H}$ and $\mathrm{OH}$ in their 


\begin{tabular}{|c|c|c|c|c|c|}
\hline & $W(\mathrm{cV})$ & $\mathrm{A}$ & $\Omega$ & $\mathrm{S}$ & $\mathrm{V}$ \\
\hline Plasmon & 21.4 & 3.725 & 0.7360 & 3 & 1 \\
$\mathrm{~A}^{1} \mathrm{~B}_{1}$ & 8.4 & 0.0302 & 0.6537 & 1.561 & 3 \\
$\mathrm{~B}^{1} \mathrm{~A}_{1}$ & 10.1 & 0.0617 & 0.6447 & 1.537 & 3 \\
$\mathrm{H}^{*} \mathrm{Lyman}$ & 17.0 & 3.49 & 1.0 & 0.3 & 1 \\
$\mathrm{H}^{*} \mathrm{H \alpha}$ & 19.0 & 0.735 & 1.0 & 0.3 & 1 \\
$\mathrm{H}^{*} \mathrm{H} \beta$ & 20.0 & 0.125 & 1.0 & 0.3 & 1 \\
$\mathrm{H}^{*} \mathrm{H} \gamma$ & 20.0 & 0.05 & 1.0 & 0.3 & 1 \\
$\mathrm{H}^{*} \mathrm{H \delta}$ & 20.0 & 0.021 & 1.0 & 0.3 & 1 \\
$\mathrm{O}^{*}, 1304 \mathrm{~A}$ & 16.0 & 0.089 & 1.25 & 1 & 3 \\
$\mathrm{O}^{*}, 8447 \mathrm{~A}$ & 17.0 & 0.22 & 4.85 & 1 & 2 \\
$\mathrm{OH}^{*}, 3064 \mathrm{~A}$ & 10.0 & 0.0537 & 1.0 & 5.35 & 1 \\
$\mathrm{OH}^{*}, 2800 \mathrm{~A}$ & 11.0 & 0.0058 & 1.0 & 2.35 & 1 \\
\hline
\end{tabular}

Table IV: Parameters for water dissociative cross-sections, Eq.(9).

ground state [10]. The second dissociation continuum aroung $9.67 \mathrm{eV}$ leads to the formation of $\mathrm{H}$ in its ground state and $\mathrm{OH}$ in a rotationally excited state [10].

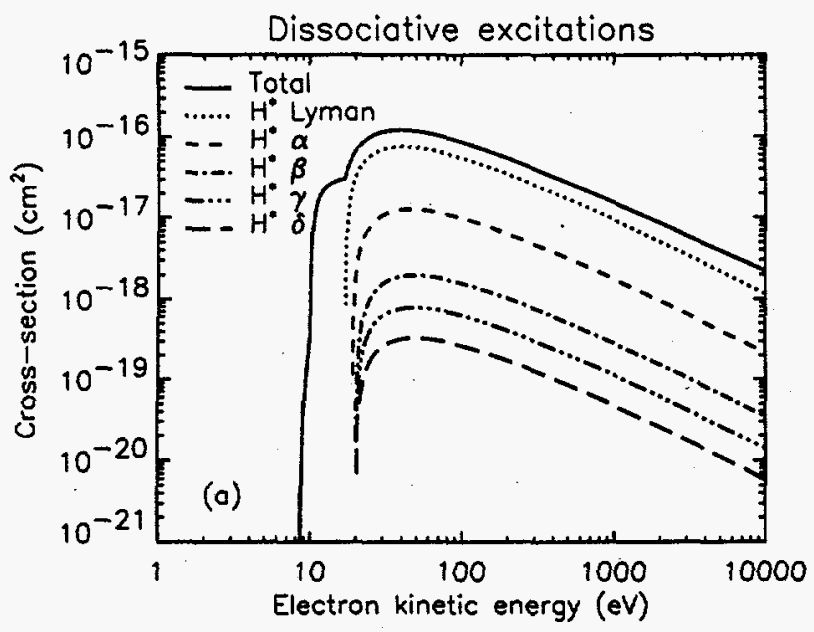

Figure 4(a): Dissociative cross-sections for water radiolysis by electrons (hydrogen products). The total cross-section corresponds to the total of the partial cross-sections shown in Figs.4(a) and (b).

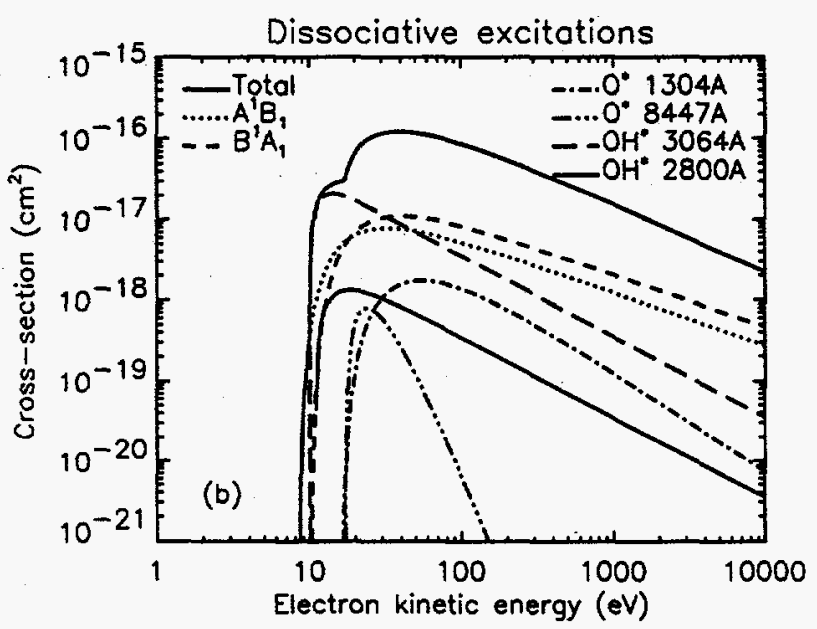

Figure 4(b): Dissociative cross-sections for water radiolysis by electrons (oxygen and hydroxyl products). The total cross-section corresponds to the total of the partial cross-sections shown in Figs.4(a) and (b).

There is a second class of dissociative excitation processes leading to the formation of excited fragments, $\mathrm{H}^{*}, \mathrm{OH}^{*}$, and $\mathrm{O}^{*}$. They are listed in Table IV together with the parameters for Eq.(9). The names of these states originates from the fact that their existence was inferred from spectroscopic measurements involving various hydrogen Lyman and Balmer lines. The cross-sections for the various dissociative processes listed here are shown in Figs.4(a) and (b).

Non-disseciative excitations: Our calculations include two sets of nondissociative excitations characteristic of the water molecule: A so-called "diffuse band" around 13.3 eV and a set of Rydberg states commonly labeled A, B, C, and D. These processes do not lead to dissociation, but they remove electron kinetic energy that might otherwise have been sufficient to cause ionization. In other words, the existence of these processes decreases the rate of ionization that would otherwise be present and lead to dissociation. The cross-section for these processes can be fitted to the analytical form Eq.(9), [14]. The parameters are given in Table V, [15]; cross-sections are shown in Fig.5.

\begin{tabular}{|c|c|c|c|c|}
\hline & $W(\mathrm{eV})$ & $\mathrm{A}$ & $\Omega$ & $\beta$ \\
\hline Diffuse bands & 14.10 & 0.0860 & 0.4535 & 3.000 \\
Rydberg (A+B) & 11.26 & 0.0142 & 0.6670 & 1.415 \\
Rydberg (C+D) & 11.93 & 0.0590 & 0.5796 & 1.813 \\
\hline
\end{tabular}

Table V: Parameters for the non-dissociative cross-sections in water, Eq.(9). For all excitations, $v=3$.

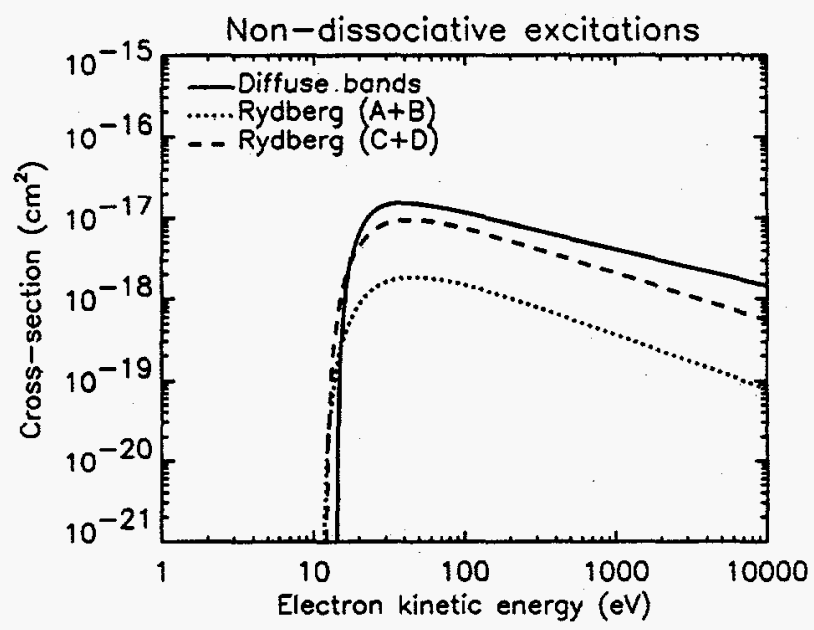

Figure 5: Non-dissociative excitation (diffuse bands and Rydberg) crosssections for electron-water collisions.

Plasmon: The existence of a very prominent plasmon mode at $21.4 \mathrm{eV}$ in liquid water is well-documented, [15]. A plasmon is a collective electromagnetic excitation, in this case, of the valence electrons of the water molecule. A plasmon in water has a lifetime of about $10^{-16} \mathrm{~s}$. It decays into $\mathrm{H}_{2} \mathrm{O}^{+}, \mathrm{OH}^{+}, \mathrm{O}^{+}$, or $\mathrm{H}^{+} 92 \%$ of the time. It decays $\mathrm{H}, \mathrm{O}$, or $\mathrm{OH}$ the rest of the time. The branching ratios for plasmon decay are given in Table VI. The water plasmon cross-section is well represented by Eq.(9) with the relevant parameters given in Table IV, [15].

Aqueous electrons: An electron which has lost so much energy that it can no longer participate in the creation of the excitations described above will end up surrounding itself with water molecules to form a kind 
of "hydration shell". The existence of aqueous electrons is now well established. They are very active, chemically-speaking and play an important role in the radiation chemistry of tracks as discussed below.

\begin{tabular}{|c|c|}
\hline Decay mode & Decay probability (\%) \\
\hline $\mathrm{H}_{2} \mathrm{O}^{+}$ & 58.29 \\
$\mathrm{OH}^{+}$ & 15.03 \\
$\mathrm{O}^{+}$ & 1.57 \\
$\mathrm{H}^{+}$ & 17.24 \\
Lyman $\mathrm{g}$ & 5.24 \\
$\mathrm{H \alpha}$ & 1.86 \\
$\mathrm{H} \beta$ & 0.39 \\
$\mathrm{H} \gamma$ & 0.21 \\
$\mathrm{H} \delta$ & 0.08 \\
$\mathrm{OI}(8447)$ & 0.08 \\
\hline
\end{tabular}

Table VI: Branching ratios for plasmon decay in water.

\section{Elastic scattering of electrons}

For electrons, we have seen that elastic scattering is important and we must therefore study one last elementary process, namely elastic scattering of charged particles

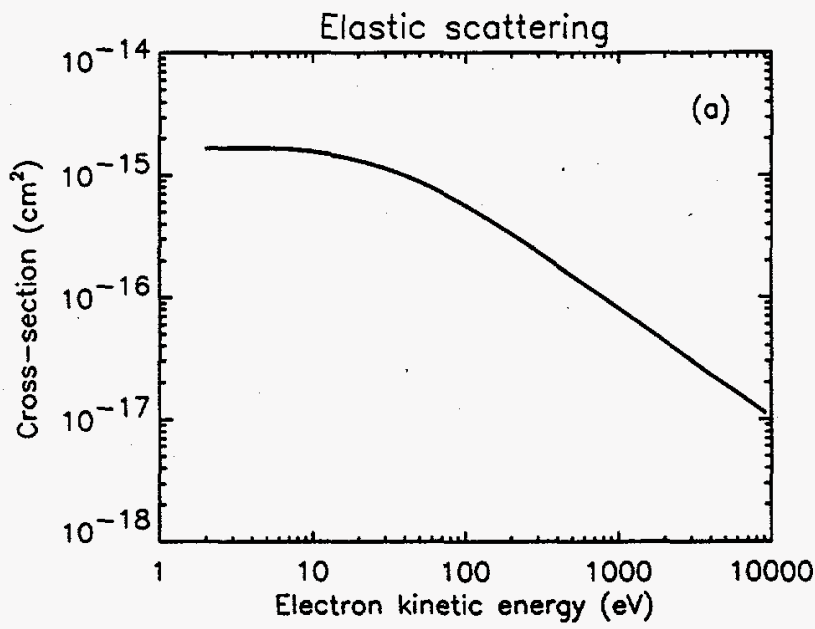

Figure 6(a): Total (Rutherford) elestic scattering cross-section of electrons in water.

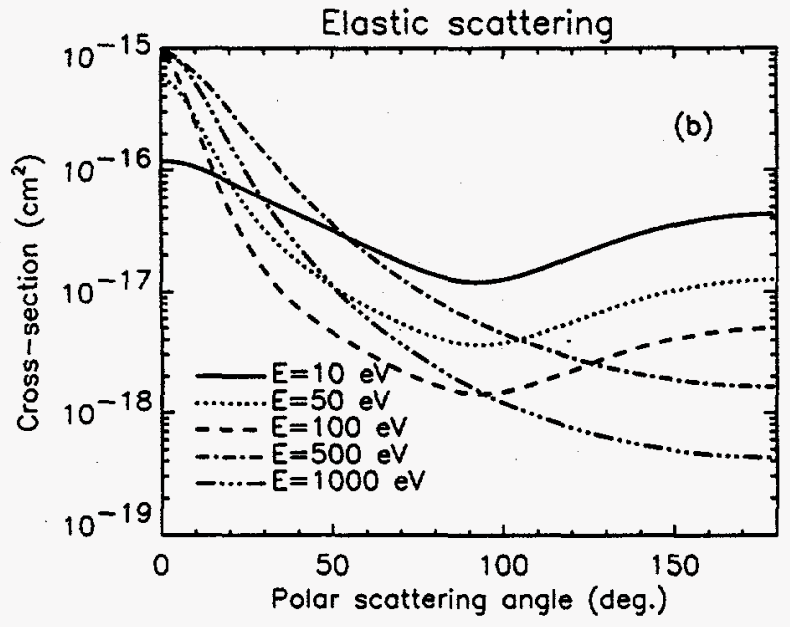

Figure 6(b): Angular dependence of the elastic scattering cross-section for $e$. in water. The Porter-Jump formula was used below $200 \mathrm{eV}$.
The elastic scattering of high-energy electrons by atoms is reasonably well described by the Rutherford cross-section including a correction term to take atomic screening into account $[14,16,17]$ :

$$
\frac{d \sigma}{d \Omega}=\frac{1}{\left(4 \pi \varepsilon_{0}\right)^{2}} \frac{Z(Z+1) e^{4}}{(1-\cos \theta+2 \eta)^{2}}\left[\frac{E+m c^{2}}{E\left(E+2 m c^{2}\right)}\right]^{2},
$$

where $E$ is the energy of the incident electron, $\theta$ the polar angle of the scattered electron, $Z$ is the atomic number of the medium, $\varepsilon_{0}$ is the permittivity of vacuum, $m c^{2}$ is the electron energy at rest, $e$ is the electron charge, and $\eta$ is the Moliere screening parameter given by [16]:

$$
\eta=\left(1.13+3.76\left(\frac{\mathrm{Z}}{137}\right)^{2} \frac{\mathrm{mc}^{2}}{2 \mathrm{E}}\right) \frac{1.7 \times 10^{-5} \mathrm{Z}^{2 / 3} \mathrm{mc}^{2}}{\mathrm{E}\left(\mathrm{E} /\left(\mathrm{mc}^{2}\right)+2\right)}
$$

Eq.(10) is easily integrated with respect to the solid angle, $\Omega$, to obtain the total elastic scattering cross-section:

$$
\sigma(E)=\frac{1}{\left(4 \pi \varepsilon_{0}\right)^{2}} \frac{Z(Z+1) \pi e^{4}}{(\eta(\eta+1))}\left[\frac{E+m c^{2}}{E\left(E+2 m c^{2}\right)}\right]^{2} .
$$

Below $1 \mathrm{keV}$, in water, elastic scattering is the major elementary process responsible for electron scattering. The Rutherford cross-section is adequate for energies down a few tens of $\mathrm{eV}$. A very low energies, the Rutherford formula becomes a poorer approximation. Several altemative formulae have been proposed. One of the most convenient formulae is that of Porter and Jump [18] because it matches the Rutherford formula asymptotically. The Porter-Jump parameters for water appear in Ref.[14]; we will not reproduce the details of the PorterJump formula here, although this cross-section was used in our calculation. Fig.6 shows the elastic scattering cross-section for electrons in water as determined by means of the Rutherford formula.

\section{Track structure and track chemistry}

So far, we have focused our attention on the elementary processes associated with the creation of secondary electrons and their interaction with water molecules. There is, however, one last concept we need to introduce to complete our modeling of radiation chemistry processes in water, namely the concept of tracks. The elementary processes described above are spatially located along tracks. These tracks correspond more or less with the trajectory of the incident particle and are strewn with secondary electron creation events corresponding to energy loss of the primary particle between two spallation events. The incident proton is also deflected by elastic scattering, but given its large velocity, this effect is small and proton tracks tend to be rectilinear over considerable distances. Secondary electrons can also form tracks. Becanse elastic scattering is more significant for electrons, electron tracks tend to be more erratic and electron tracks form entities described in the radiation chemistry literature by such evocative names as spurs and blobs [19]. Along these tracks -proton and electron tracks- the elementary phenomena discussed above take place with frequencies determined by the corresponding cross-sections. It is important to determine the spatial structure of tracks because the water radiolysis products are produced in the immediate vicinity of the track. Immediately after their formation, which takes place on the scale of 1 ps or so, these primary radiolysis products will diffuse and react with each other, with water molecules, or with any atom or molecule present in their immediate vicinity. Diffusion and track reactions is a rapid process, lasting of the order of a few $\mu \mathrm{s}$. However, in the small volume comprised by these tracks, the concentration of reactive species can be extremely high. The outcome of the "track reactions" phase depends very much on the initial distribution of the primary radiolysis products, i.e., on the detailed structure of the track.

A large number of studies have been and continue to be devoted to the study of the structure of tracks $[4,19,20]$. We will adopt a simple model developed by Schwartz to describe track structure [21]. This model, when used with deterministic models of radiation chemistry, seems to produce results in very good agreement with more sophisticated microscopic calculations [22]. In the traditional deterministic approach to the kinetics of track structures, the time and space evolution of each chemical species present in the system is determined by a set of coupled partial differential equations for the concentration $C_{1}$ of the $i$-th species [21,22]: 


$$
\frac{\partial C_{i}}{\partial t}=D_{i} \nabla^{2} C_{i}-\sum_{j} k_{i j} C_{i} C_{j}+\sum_{j} \sum_{k} k_{j k} C_{j} C_{k},
$$

where $D_{i}$ is the diffusion coefficient of the $i$-th species, $k_{i j}$ is the rate constant for the reaction between the $i$-th and $j$-th species. In Eq.(13) the first term in the right-hand side is a classic diffusion term, the second term gives the rate at which the $i$-th species disappears from the system. the last term is the rate of production of the $i-t h$ species from the reaction of $j$ with $k$. (This is the set of equations solved by FACSIMILE. See below.) Low energy electron spurs tend to be more or less spherical in shape; higher energy tracks are more or less cylindrical. Schwartz recognized this fact by assuming that the initial spatial distribution of the chemical species is spherically or cylindrically symmetric, as the case may be. More precisely, he made the assumption that the initial distribution of reactants is Gaussian; in the case of a spherically symmetric spur, for instance, the initial distribution of type i molecules is:

$$
C_{i}(r)=\frac{N_{i}}{\left(2 \pi \sigma_{i}\right)^{3 / 2}} \exp \left(-\frac{r^{2}}{2 \sigma_{i}^{2}}\right)
$$

where $N_{1}$ is the number of molecules of type $i$ in the spur, and $\sigma_{t}$ is a

\begin{tabular}{|c|c|}
\hline Reaction & Rate constant $\left(\mathrm{mol}^{-1} \cdot \mathrm{s}^{-1}\right)$ \\
\hline 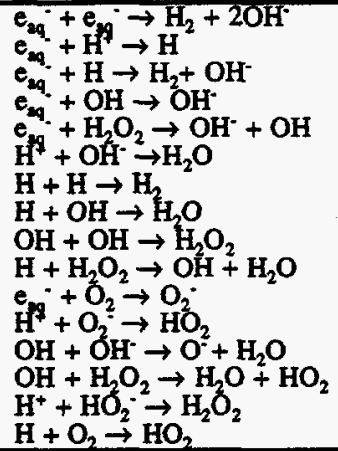 & $\begin{array}{c}0.55 \times 10^{10} \\
2.3 \times 10^{10} \\
2.5 \times 10^{10} \\
3.0 \times 10^{10} \\
1.1 \times 10^{10} \\
14.3 \times 10^{10} \\
0.78 \times 10^{10} \\
2.0 \times 10^{10} \\
0.55 \times 10^{10} \\
0.009 \times 10^{10} \\
1.9 \times 10^{10} \\
3.8 \times 10^{10} \\
1.3 \times 10^{10} \\
5 \times 10^{7} \\
5 \times 10^{10} \\
2.1 \times 10^{10}\end{array}$ \\
\hline
\end{tabular}
characteristic length (that will be determined later).

Table VII: Main chemical reactions considered in our model of water radiolysis and their rate constants.

The last piece of information we need is a set of chemical reactions that can take place between the radiolysis products, as well as the corresponding rate constants. These reaction have been studied extensively by radiation chemists. The most important ones are reproduced in Table VII where the rate constant for the reaction is also listed. The diffusion coefficients of the various chemical species involved in track reactions are listed in Table VIII.

\begin{tabular}{|c|c|}
\hline Species & Diffusion coefficient $\left(\mathrm{m}^{2} \mathrm{~s}^{-1}\right)$ \\
\hline $\mathrm{e}_{19}^{-}$ & $4.5 \times 10^{-9}$ \\
$\mathrm{H}^{-}$ & $9.0 \times 10^{-9}$ \\
$\mathrm{H}$ & $7.0 \times 10^{-9}$ \\
$\mathrm{OH}$ & $2.8 \times 10^{-9}$ \\
$\mathrm{H}_{2}$ & $5.0 \times 10^{-9}$ \\
$\mathrm{OH}^{-}$ & $5.0 \times 10^{-9}$ \\
$\mathrm{O}_{2}^{-}$ & $2.1 \times 10^{-9}$ \\
$\mathrm{HO}_{2}$ & $2.0 \times 10^{-9}$ \\
$\mathrm{H}_{2} \mathrm{O}_{2}$ & $2.2 \times 10^{-9}$ \\
\hline
\end{tabular}

Table VIII: Diffusion coefficients of the main water radiolysis products.

\section{Computational Methodology}

\section{Computercodes}

Before we proceed to a more detailed discussion of the computational methodology we developed to calculate water radiolysis in the spallation radiation environment, we give a brief description of the computer codes used in our calculations.
The LAFET Monte Carlo code is a radiation transport code [23]. It uses the Bertini model to determine the nuclear interaction of high-energy nucleons with matter. The user can specify an arbitrarily complex 3-D geometry for the system to be studied. Cells are region of space bounded by surfaces and filled with a material or mixture of materials. LAHET will also provide information on the creation of photons, neutrons, pions, muons, electron, and positrons, as well as recoiling ions. Photons and neutrons with energies below $20 \mathrm{MeV}$ are saved in a file for transport with the radiation transport code MCNP.

MCNP is also a Monte Carlo radiation transport code [24]. It is used transports low energy neutrons, photons, and electrons. It uses the same computer model of the system under consideration as LAHET, and makes use of ENDF/B-VI cross-sections to determine the interaction of neutrons with matter. Both LAHET and MCNP can provide microscopic information such as individual particle trajectory and velocity, as .well as fluxes, currents, reaction rates, and energy deposition. The use of LAHET and MCNP allows us to completely characterize the radiation environment of a spallation neutron source. LAHET and MCNP were written at and are available from Los Alamos National Laboratory.

The last computer code used, FACSIMIIE, is a general-purpose code to study steady-state and time-dependent processes, particularly chemical reactions with diffusion and/or advection [25]. After generating tracks from the information provided by LAHET and MCNP, we are left with the coordinates of the various primary radiolytic products. This information, together with a set of diffusion constants and possible chemical reactions with their rate constants, is used as initial conditions by FACSIMILE to determine the evolution in time of the concentration of radiolytic products and the end products of the track reactions by solving the resulting system of reaction-diffusion differential equations for the species concentration. The rate constants for the chemical reactions governing water radiolysis are known accurately. Basic water radiation chemical reactions and all their parameters were kindly provided to us by Prof. Jay LaVerne (University of Notre Dame Radiation Laboratory).

\section{Methodology}

We now describe in more detail the various steps involved in a complete calculation of water radiolysis for a spallation neutron source.

- Set up a computer model of the source: for LAHET and MCNP. Use these models to calculate the radiation environment and the energy deposition in water due to the various particles present. Ultimately, the energy deposition calculated by LAHET and MCNP reflects the amount of energy spent creating secondary electrons. If the radiation field varies spatially it is important to calculate the local radiation field at several locations. For instance, as the proton beam penetrates into the target, the radiation environment changes as fewer, less-energetic protons are available for spallation. It follows that the nature of the radiation field at the upstream and downstream ends of the target will be quite different.

- Calculate one or several tracks at desired locations: This can be performed concurrently with the calculation of the radiation field To this effect, we have added a set of subroutines to LAHET and MCNP to generate the secondary electrons as protons or other particles are transported in the system. The location and velocity vector of each secondary electron is saved in a file. This information is then passed on to a small program that uses this file and the cross-section information described in the preceding section to generate more secondary electrons or various events such as vibrational excitations, dissociative and non-dissociative excitations, or ionization. Electrons are transported until their energy drops below the point where they are incapable of generating any kind of excitation. At this point, the user has the choice between two distinct possibilities. The location and nature of each event can be saved, to be used as an input for Monte Carlo codes of radiation chemistry. This results in the storage of extremely large files large files and is therefore limited to small track segments. Furthermore, the Monte Carlo codes developed by radiation chemists are slow and provide information that is too detailed for our purpose here. For this reason, we prefer the alternative, which is to determine, as the various events are generated, the parameters of the Schwartz model (or any such model) for the track structure and let FACSIMILE generate initial conditions based on this model. If several tracks are included for one location in the system, it is possible to take an average over tracks to calculate an "average" track, or calculations can be performed for individual tracks. 
- Solve the reaction-diffusion problem with FACSIMILE: based on the set of reactions, diffusion constants, and initial conditions determined previously. FACSIMILE offers a great deal of flexibility: The initial conditions can be varied easily (e.g., different track model); physical parameters such as temperature can be varied; chemical reactions can be added. This latter point is particularly interesting as it allows one to take into account the presence of "scavengers", chemical species added to the water that react with the radiolysis products to modify the track chemistry. The final result provided by FACSIMILE in our calculation is the so-called G-value corresponding to each chemical species as a function of time. It is simply the number of molecules, atoms, or ions present in the medium per $100 \mathrm{eV}$ of energy deposited in the medium.

\section{Example: The APT Target Station}

\section{The API project}

In the APT project, a $1.7 \mathrm{GeV}$ proton beam from a linear accelerator is used to bombard a (stopping-length) tungsten target and produce neutrons via spallation reactions in tungsten. The neutrons are then moderated by a heavy-water blanket surrounding the target. The moderated neutrons are then captured by ${ }^{3} \mathrm{He}$ gas flowing in tubes throughout the water blanket. Tritium is produced via the $n\left({ }^{3} \mathrm{He}, \mathrm{p}\right) \mathrm{T}$ reaction. The target consists of a series of rod bundles with water flowing between individual rods. A main concern in the project is the corrosion and radiation-enhanced corrosion of the irradiated, watercooled tungsten rods.

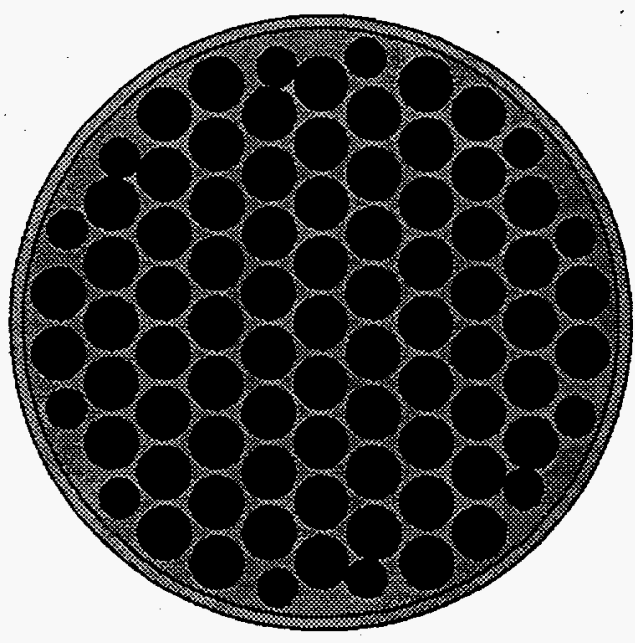

Figure 7: Cross-section of a tungsten rod bundle. (Fifteenth rung of the first ladder in the APT target station.) The bundle consists of 73 larger tungsten rods and 12 smaller ones contained in a stainless steel tube. Water flows longitudinally along the rods, in the space between them. Dimensions are given in the text.

Figure 7 shows a detail of the LAHETMCNP computer model for the APT system, namely a rod bundle in the first "ladder" of target rod bundles. The rod bundles are all alike; they are arranged deeper into the system, along the path of the beam. For the sake of illustrating the computational methodology described above to study water radiolysis, we will focus on this first rod bundle. As shown in Fig.7, the bundle of tungsten rods is contained in a stainless steel (SS304) tube $3.75 \mathrm{~cm}$ in diameter. The individual rods are $0.318 \mathrm{~cm}$ in diameter for the 73 larger rods, and $0.235 \mathrm{~cm}$ in diameter for the 12 smaller ones. The tube and the rods are $22 \mathrm{~cm}$ long. Water flows longitudinally, between the rods and along them, from one end of the stainless steel tube to the other end. The total volume of water in a tube is $84.8 \mathrm{~cm}^{3}$. The volume fraction of tungsten inside the tube is about $62 \%$.

\section{Energy deposition}

Our first step consists in determining the energy deposition in water due to the various particles present in the radiation field. Table IX summarizes the results of a LAHET/MCNP calculation.

\begin{tabular}{|c|c|c|}
\hline Particle & Energy deposited (keV) & \% of tota \\
\hline $\mathrm{p}$ & 85.9 & 68.3 \\
$\mathrm{n}$ & 18.8 & 14.9 \\
$\gamma^{\gamma}$ & 3.5 & 2.8 \\
$\pi^{+}, \pi$ & 4.7 & 3.7 \\
$\mu^{+}, \mu^{-}$ & 0.3 & 0.2 \\
$\mathrm{~d}^{-}$ & 2.4 & 1.9 \\
$\mathrm{t}$ & 2.0 & 1.6 \\
${ }^{3} \mathrm{He}$ & 0.4 & 0.3 \\
${ }^{4} \mathrm{He}$ & 3.7 & 2.9 \\
Nuclear recoils & 2.1 & 1.7 \\
$\mathrm{e}^{+}, \mathrm{e}^{-}$ & 2.0 & 1.6 \\
Total & $\mathbf{1 2 5 . 8}$ & $\mathbf{1 0 0 . 0}$ \\
\hline
\end{tabular}

Table IX: Energy deposition in the cooling water of the APT rod bundle.

It is rather clear that as far as the first rod bundle is concerned, protons are essentially responsible for energy deposition, and, therefore, for the creation of secondary electrons. The total amount of energy deposited in the water is sizeable. The "footprint" of the proton beam is $16 \mathrm{~cm} \mathrm{x} 160$ $\mathrm{cm}$. It is centered on the target. So, effectively, a smaller fraction (16 $\mathrm{cm} / 22 \mathrm{~cm}=0.727$ ) of the volume of water is directly exposed to the beam. APT relies on a proton beam current of $100 \mathrm{~mA}$. A simple calculation shows that at this current and assuming a uniform beam profile, $1.46 \times 10^{16}$ protons hit our rod bundle each second. Given a total energy deposition of $161.9 \mathrm{keV}$ per proton incident on the entire target, this correspond to an energy deposition of $379.5 \mathrm{~J}$ per second or an average power of $379.5 \mathrm{~W}$ in the water. This corresponds to an average power density in the water of about $4.5 \mathrm{~kW} /$.

\section{Tracks}

The second step in our calculation consists in generating tracks. The track structure for a $1.7 \mathrm{GeV}$ proton is relatively simple. Elastic scattering for a $1.7 \mathrm{GeV}$ proton is negligible and the proton travels essentially in a straight line between two spallation events. The mean free path to spallation for a $1.7 \mathrm{GeV}$ proton in water is roughly $60 \mathrm{~cm}$, whereas the mean free path for the creation of secondary electrons is roughly $1 \mu \mathrm{m}$. The several orders of magnitude difference between the mean free path for spallation and the mean free path for the creation of secondary electrons implies that along the proton track, there will be many secondary electrons created between any two spallation events.

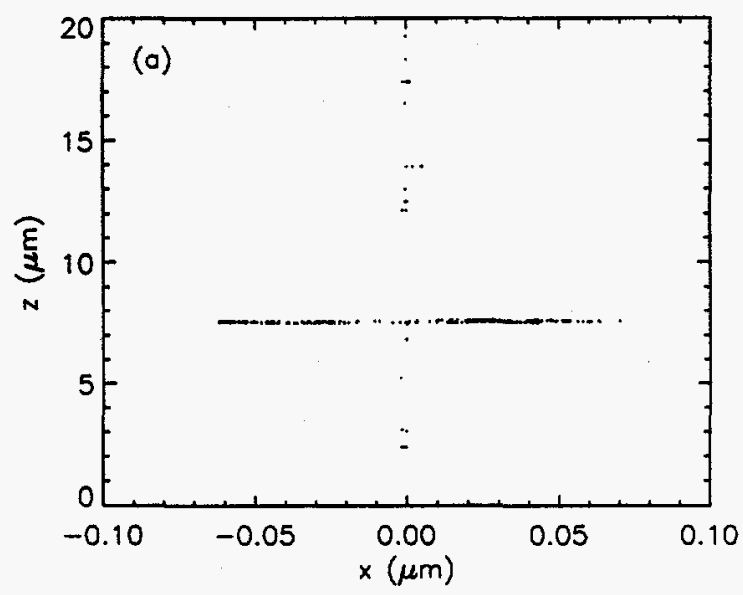

Figure 8(a): Segment of a track of a $1.7 \mathrm{GeV}$ proton in water in the APT rod bundle. Each dot represents an event such as ionization, excitation, etc. The proton propagates along the $\mathrm{z}$-axis. At $\mathrm{z}=7.5 \mu \mathrm{m}$ or so, a secondary electron with energy $4.2 \mathrm{keV}$ is created by the proton (see Fig.8(b)). 


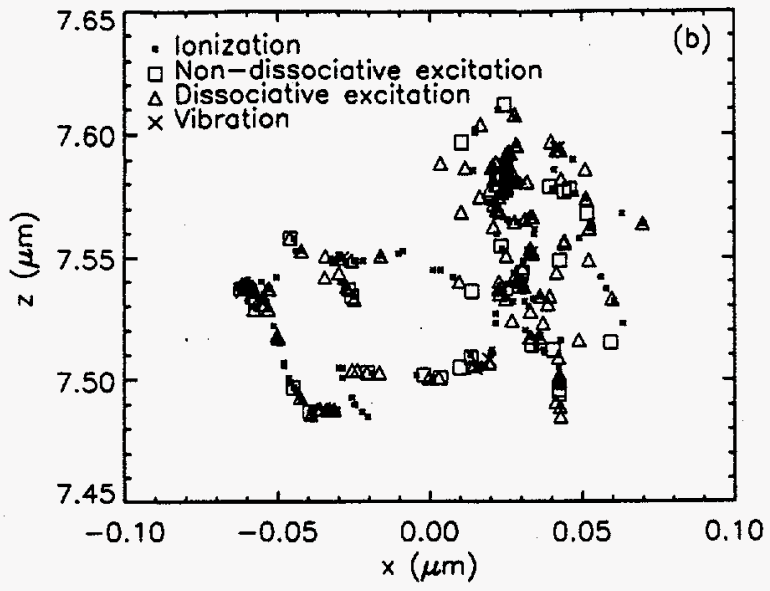

Figure 8(b): Detail of the $4.2 \mathrm{keV}$ secondary electron track discussed in the caption of Fig.8(b).

Given the volume of water in the rod bundle and the dimensions of the latter, the proton beam traverses the equivalent of almost $1 \mathrm{~cm}$ of water as it passes through the rod bundle. If the mean free path for the creation of secondary electrons is approximately $1 \mu \mathrm{m}$, each protons creates on average $10^{4}$ secondary electrons. The secondary electrons will, occasionally, have enough kinetic energy to initiate their own track. Along these tracks, some newly created electrons may still have enough kinetic energy to create tracks branching off the main track, Fig.8(a). (Notice the difference in the $x$ and $z$ scales in Fig.8(a).) At some point, however, the electrons will no longer have enough kinetic energy to create rectilinear tracks. They will be strongly (elastically) scattered while losing the remainder of their kinetic energy by creating all kinds of low-energy excitations. These "end-of-the track" blobs tend to be more or less spherical. Fig.8(b) shows the details of the long "side track" in the middle of Fig.8(a); it is a projection of the blob on the $(x, z)$-plane. This event corresponds to a high-energy deposition event of $4.2 \mathrm{keV}$, a quite rare event.

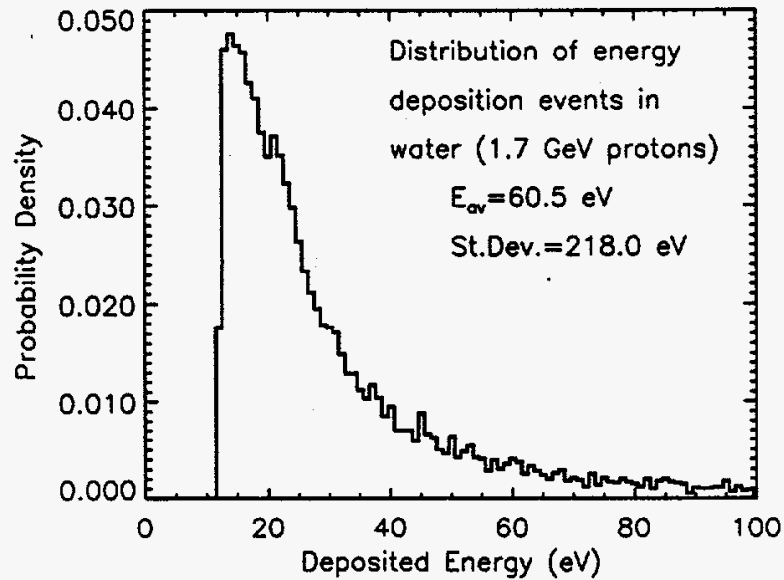

Figure 9: Distribution of energy deposition events for a $1.7 \mathrm{GeV}$ protons in the APT rod bundle.

It is easy to divide the tracks and blobs into individual entities, and collect information such as $\sigma_{1}$ and $N_{1}$ (the parameters of the Schwartz model) as well as the deposited energy associated with each blob or track segment. Figure 9 shows the statistical distribution of energy deposition events in a typical track for a $1.7 \mathrm{GeV}$ proton. The average energy deposition event deposits $60.5 \mathrm{eV}$. The distribution is very asymmetric and its mode (14 $\mathrm{eV}$ or so) differs significantly from the average.

\section{G-yalues}

For a given deposited energy, we calculated the Schwartz parameter of typical track or blob and used that information as input for FACSIMILE. FACSIMILE solved the reaction-diffusion problem for the track or blob and produced a series of G-values as a function of time G-values are shown in Fig. 10 for a $50 \mathrm{eV}$ energy deposition event for a number of radiolysis products. As mentioned in the introduction, the concentration of short-lived products can be very high, albeit for a short period of time.

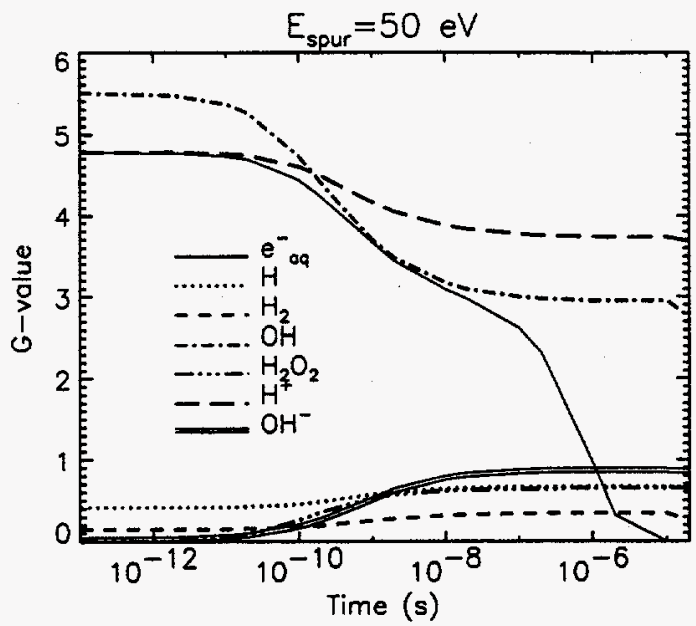

Figure 10: Average G-values as a function of time for a number of water radiolysis products for a $50 \mathrm{eV}$ energy deposition event. in the APT rod bundle.

A total G-value curve can be generated by weighting the various sets of G-value curves corresponding to different values of the deposited energy with the curve shown in Fig.9. This is shown in Fig.11 for $\mathrm{H}_{2} \mathrm{O}_{2}$. The production of hydrogen peroxide is quite significant, $G=0.5$ molecules per $100 \mathrm{eV}$ of deposited energy. For our rod bundle, this corresponds to the formation of $0.4 \times 10^{-3} \mathrm{~mol}$ of $\mathrm{H}_{2} \mathrm{O}_{2}$ per $\mathrm{s}$ or $1.6 \mathrm{~mol}$ of $\mathrm{H}_{2} \mathrm{O}_{2}$ per hour (54 grams per hour). This is for one rod bundle and includes only the contribution of the proton component of the radiation field! (We assumed a current on the entire target of $100 \mathrm{~mA}$ and a beam spot size of $16 \mathrm{~cm} \times 160 \mathrm{~cm}$.

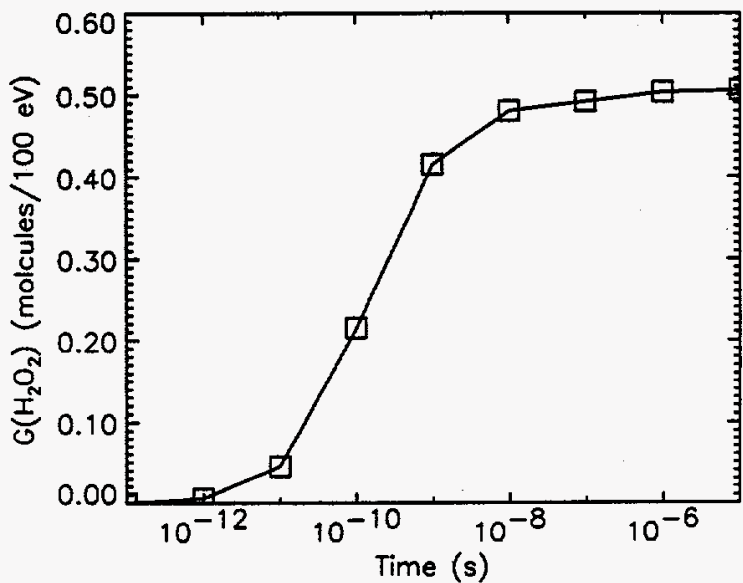

Figure 11: Total (all energy deposition events) $\mathrm{G}$-value for $\mathrm{H}_{2} \mathrm{O}_{2}$ in the APT rod bundle.

The cooling water flows continuously in the APT system and the amount of $\mathrm{H}_{2} \mathrm{O}_{2}$ calculated above is constantly getting diluted in a much larger 
volume of water. It remains true that a generation rate (per rod bundle, for protons) of 54 grams per hour is rather large and cause for concern as far as corrosion is concerned.

FACSIMILE is also capable of giving information on the distance radiolysis products diffuse during the track reaction. From this and a detailed knowledge of the geometry of the water-tungsten interface, one can estimate the number of short-lived species that might react directly with the tungsten surface. This, and additional results, will be presented elsewhere.

\section{Conclusion}

The set of basic information (nature and rate of formation of radiolysis products; rate of collision with surfaces; reaction rates with scavengers should prove useful to corrosion chemists and engineers, to understand the nature of the corrosion process, predict its rate, and mitigate its effects. The availability of a computational tool makes it convenient to recalculate basic corrosion parameters whenever a change is made to the target design. In particular, it is easy to study the impact on corrosion of changes such as a change in beam energy, a change in target or structural material, a change in the cooling water temperature, etc. This, of course, assumes that this computational tool bas been extensively benchmarked. Simplifications were introduced at various stages in the modeling, in all computer codes, models and cross-section data. While many of those have been used successfully before, it remains to be seen how the entire code performs. To this effect, we have started a series of basic experimental studies at Los Alamos. We are using ultra-fast optical spectroscopic techniques (resonant Raman scattering, optical absorption) to study the radiation and corrosion chemistry of a protonirradiated water/metal interface. Besides providing us with useful information to benchmark the computational tool described above, it also allows us to examine in situ the formation of corrosion product in an irradiated metal. Preliminary results of these studies will be reported in another paper during this conference.

One last word of caution is in order. Other physical and electrochemical phenomena influence the rate of corrosion of an irradiated metal. Radiation damage to the metal can lead to bulk and surface modifications that inhibit or enhance corrosion. The electrochemistry of an irradiated electrode is far from being understood. The addition of reducing agents to the cooling water or the presence of other scavenging impurities can all contribute to a modification of corrosion rates. Nonetheless, water radiolysis is a major player in the corrosion of materials at nuclear facilities.

\section{Acknowledoments}

The authors are grateful to J. LaVerne for assistance with various radiation chemistry problems. We thank G. Willcutt and R.B. Kidman for providing information on the APT target system. This work was supported by the U.S. Department of Energy.

\section{References}

1. The litterature on this topic is too vast to mention here. Recent work in this area appears in T.K. Yeh, D.D. MacDonald, and A.T. Motta, Nucl.Sci.Eng., 121, 468 (1995) where additional useful references can be found.

2. J.W.T. Spinks and RJ. Woods, "An Introduction to Radiation Chemistry", (J.Wiley \& Sons, Inc., New York, 1990); A.O. Allen, "The Radiation Chemistry of Water and Aqueous Solutions", (D.Van Nostrand Company, Inc., Princeton, 1961); sec also Refs. 4, 19, and 20.

3. R. Serber, Phys.Rev., 72, 1114 (1947).

4. H.G. Paretzke, in "Kinetics of Nonhomogeneous Processes", G.R. Freeman, Ed., (J.Wiley \& Sons, New York, 1988), p.89.

5. C.B. Opal, E.C. Beaty, and W.K. Peterson, At Data, 4, 209 (1972) C.B. Opal, W.K. Peterson, and E.C. Beaty, J.Chem.Phys., 55, 4100 (1971).

6. A.E.S. Green and T. Sawada, J.Atm.Terr.Phys., 34, 1719 (1972). See also B. Grosswendt and E. Waibel, Nucl.Instr.Meth 155, 145 (1978 and M. Zaider, D.J. Brenner, and W.E. Wilson, Rad.Res., 95, 231 (1983).
7. M.J. Berger, in the Proceedings of the "Second Symposium on Microdosimetry", H.G. Ebert, Ed., (Commission of the European Communities, Brussels, 1969), p.541.

8. J.D. Garcia, Phys.Rev., 177, 223 (1969). See also Appendix I in J.H. Miller and E.S. Green, Rad.Res., 54, 343 (1973).

9. S.M. Pimblott, J.A. La Verne, A. Mozumder, and N.J.B. Green, J.Phys.Chem., 94, 488 (1990); S.M. Pimblott, A. Mozumder, J.Chem.Phys., 95, 7291 (1991)

10. J.J. Olivero, R.W. Stagat, and A.E.S. Green, J.Geophys.Res., 77, 4797 (1972)

11. S.T.S. Dayashankar and A.E.S. Green, Int.J.Quantum Chem. (Symposium), 20, 547 (1986). See also Ref.17.

12. G. Seng and F. Linder, J.Phys. B, 9, 2539 (1976).

13. S. Trajmar, W. Williams, and A. Kupperman, J.Chem.Phys., 58, 2521 (1973).

14. M. Zaider, D.J. Brenner, and W.E. Wilson, Rad.Res., 95, 231 (1983).

15. G.J. Kutcher and A.E.S. Green, Rad.Res., 67, 408 (1976).

16. B. Grosswendt and E. Waibel, Nucl.Instr.Meth., 155, 145 (1978).

17. M.A. Hill and F.A. Smith, Radiat.Phys.Chem., 43, 265 (1994),

18. H.S. Porter and F.W. Jump, Computer Science Corp. Report CSC/TM-78/6017 (1978)

19. J.L. Magee and A. Chatterjee, in "Kinetics of Nonhomogeneous Processes", G.R. Freeman, Ed., (J.Wiley \& Sons, New York, 1988), p.171.

20. I.G. Kaplan and A.M. Miterev, Adv.Chem.Phys., Vol.LXVIII, 255 (1987).

21. H.A. Schwartz, J.Phys.Chem., 73, 1928 (1968).

22. S.M. Pimblott and J.A. La Verne, Rad.Res., 122, 12 (1990).

23. R.E. Prael and H. Lichtenstein "User Guide to LCS: The LAHET Code System", LA-UR-89-3014 (1989). This document is available on the World-Wide Web at http://www-xdiv.lanl,gov/XTM/lcs/lahetdoc.html

24. JF. Briesmeister, Editor, "MCNP - A General Monte Carlo N. Particle Transport Code", Los Alamos National Laboratory Report LA12625-M (1993).

25. FACSIMILE v3.0 User's Guide (AEA Technology, Harwell, 1994). 